cartographic techniques

\section{Pre-Press and Technical Support Technician}

Mark Loppnow

Image Systems, Inc.

N94 W14530 Garwin Mace Drive

Menomonee Falls, WI 53051

mloppnow@imagesystems.biz

\section{Introduction}

Creating effective, high quality maps takes a great amount of research in terms of map content, design, and production. Cartographers prepare some of the most complex digital files using a broad array of the functions in Illustration, Page Layout and GIS software. Because maps are commonly complex and require high quality output, cartographers often present challenging graphic files to their printer or publishers. From the perspective of a pre-press technician, the following topics relate to the preparation of graphic files for printing and should be of interest to cartographers:

1. File formats that are acceptable to the printer

2. Font formats, as well as a little font history

3. Color trapping for the printing industry

\section{FILE FORMAT DESCRIPTIONS}

There are several acceptable file formats that may be submitted to a printer for final publication. This section discusses these formats in terms of broader categories and conveys issues about these varying formats as they relate to publishing maps.
Customer's Software Application's

Native Format

Specifically these include:

QuarkXPress by Quark Inc.; Pagemaker, InDesign, and Illustrator by Adobe Systems Inc.; Microsoft ${ }^{\circledR}$ Office ; CorelDRAW Graphics Suite by COREL ${ }^{\mathrm{TM}}$ Corporation, Freehand MX by Macromedia Inc., etc.). The biggest advantage to using these formats is that the printing company or prepress department can, with cooperation of the cartographer, easily make last-minute changes to your files. These changes include modifying panels for special folding requirements, resaving files to other formats (EPS for example), or other problems with the file. By including these files when working with a printer, cartographers can save time because alterations can at times be made over the phone after review of the first proof. Be wary of printers that may make inadvertent and innocent changes or improvements to the design of the document that may "better fit" their production requirements. The printer should note and get approval for all changes that will be made to the document, prior to making those changes. Even as experience is gained in working with a particular printer it is important to have a well defined process for making changes so that surprises can be avoided.

The printer or prepress department can make the final files for printing in the formats they may prefer. These formats may favor specialized raster image processors (RIPs) rather than native EPS, Postscript, PDF, etc. The process requires hardware or software that interprets the PostScriptbased files and converts them into high-resolution bitmapped graphics and images. These images are required for proofing, plating, and printing. "Bitmaps" are computer graphics composed of a defined pattern of colors of dots or pixels that make up a picture. It is a good idea to check with the printer to insure that the files used for printing are the same files RIP'd for proofing. These are the same files that should have been approved at the proofing stage of the process. If they are not, it is strongly advisable to attend the press-check to re-evaluate the entire printing form again.

If the printer is being supplied with multiple copies of the document in different formats it is absolutely critical that each copy of the map is correct. It is also a good idea to make sure the printer has the same versions of software that were used to produce the document.

All fonts must be collected and provided to the printer with no conflicts. Because some methods of copying fonts are not foolproof, test the media that is being provided to the printer.

It is important that the printer or prepress department is familiar with the use of the software applications that were used to create the map. This is especially important if the printer is not in the same geographic location as the cartographer. Several weeks can be lost if the printer is required to purchase and gain sufficient expertise to edit a map in its native file format.

Some software applications, like Microsoft ${ }^{\circledast}$ products or many desktop GIS software do not support the range of color models and publishing options that are optimal for publication. While the output of these software packages can be published, it may require substantial additional effort.

\section{PostScript Based Formats}

Encapsulated PostScript (EPS) Files: In many cases EPS files are smaller and therefore easier for 
electronic transfer; although this depends heavily on the software that creates the EPS file. Because EPS files may include and embed all images, they can be easier to manage when it comes to supplying the printer with the files needed to complete your project. Because EPS is just a device-independent form of the PostScript language, it can be desirable to use because it allows a wider range of design features to be included and interpreted, like gradients. However, EPS still requires care in providing fonts to the printer because they often cannot be embedded into the EPS file. For large maps or maps that contain high-resolution images, EPS files can still be very large files. Finally, EPS files are very difficult to edit or alter, which can be a blessing or a curse.

Portable Document Format (PDF) Files: The size of PDF files can be controlled by using any of several levels of compression. This depends on which software you are using to produce the PDF files. No fonts or images files need be managed by the mapmaker if the PDF is prepared by embedding that information, which also compresses it. Furthermore, PDF files may be produced such that some degree of editing is possible, particularly with Adobe products.

PDF's can also be used for soft proofing (on monitor), as well as the ability to prepare print quality files for hard proofing and printing. See Burnett, 2004 for details on the pros and cons of soft proofing.

Publishing maps using the latest version of PDF, which is 1.6 is recommended because many past issues with PDF were improved and most printers now have the expertise to handle even the most advanced of design elements.

\section{File Format Strategies}

Problems with PDF's are very rare and therefore it is the pre- ferred file format, but problems can still occur. Ultimately the files for most maps will need to be RIP'd. Embedded images are one item that can cause the RIP not to work. Thus, the best practice for embedding images into the files given to a printer, is to embed TIFF or EPS images into the map. Using other formats, particularly the native formats from various software packages can cause the file not to RIP. Also using LZW compression can be problematic. Another trick printers use to get files to RIP is to use Adobe Acrobat to convert the file from PDF to EPS and potentially back again. Effectively this homogenizes the file; it is something that most map makers can try as well to gauge the effects.

There are also heavier or more industrial strength remedies for files that will not RIP. Some RIPs, such as Rampage, usually can identify the problem file(s) contained in the PDF, and either the printer's prep deptartment will fix it, or the cartographer will be advised on a course of action to correct the file problem(s). Other systems are $\mathrm{DaVince}$ Tools by DaVince, Prinergy by Creo Inc., etc.

To deliver files to a printer, the accepted method for electronic file transfer for proofing, followed by printing is FTP (file transfer protocol). The advantages for using FTP include PC and MAC compatibility, speed of transfer, and FTP is available to everyone and easy to use.

\section{FONT FORMATS}

As mentioned earlier it is often necessary to include additional files like fonts when providing printer with the files they will need to publish a map. By understanding types of fonts and some of their basic properties it will be easier to provide a printer with what they need.

\section{PostScript Fonts}

PostScript fonts were invented by Adobe Systems Inc. and the Type 3 font was introduced in 1984 with the MacIntosh computer, PostScript language, Aldus PageMaker and the Apple Laserwriter printer. Prior to that desktop publishing was virtually nonexistent as computers were mostly used for rudimentary word processing and spreadsheets. Typically one font resided on the computer, while expensive computers had several fonts. However, nobody knew how the typed words would look until they were printed, because the on screen representation was a bitmap or scaled multiples of bitmaps; the true printer font or fonts resided only on the desktop printer.

Type 1 fonts appeared in 1989 and Adobe Systems Inc., along with other manufactures were able to produce type 1 fonts as the licensing was less restrictive. Type 1 fonts are more compact than Type 3 fonts and use special rasterization algorithms to produce better looking output at small sizes and low resoutions. Type 1 fonts include hints, which further characterize the shape of each character and are used as instructions to insure consistency of appearance (Adobe Systems Inc.).

\section{TrueType Fonts}

TrueType fonts were invented by Apple Computer, Inc. and introduced in 1991. TrueType fonts have several advantages over Postscript Type 1 and Type 3 fonts. First is characters are based on Unicode, which is a character coding system designed to support the worldwide interchange, processing, and display of written texts of diverse languages of the modern world. Second, TrueType fonts use a single font for screen display and print, as opposed to Postscript, which requires a 
Screen font for display, and a Printer font for printing. The software that displays or draws TrueType fonts on a computer screen has special code to create type face effects like Bold, Italic, etc. Third, TrueType fonts allow for greater complexity in hinting of characters for on screen display and low-resolution output. This is increasingly important as web use grew in the late 1990's.

\section{OpenType Fonts}

OpenType fonts were invented by Microsoft Inc., and Adobe Systems Inc., and were introduced in 1997. OpenType fonts were developed to address the incompatibility problems of Postscript and TrueType fonts across Macintosh and Windows operating systems. OpenType fonts can contain either PostScript or TrueType font outlines (font names are suffixed otf or .ttf respectively) and each weight is supplied as a single, cross-platform file. The .otf format seems to work best for cross-platform use. Like TrueType, OpenType is based on Unicode and therefore may contain as many as 65,000 glyphs for many languages, and may include: oldstyle figures, true small capitals, fractions, swashes, and a full range of ligatures.

While OpenType fonts are clearly the strongest choice from an economic and file management standpoint, the old adage of don't fix something that isn't broken still definitely applies.

One critical issue with fonts is to make sure the version of the typeface is identical. For instance archives at Image Systems, Inc. show there are four different versions for the same font, Helvetica. When publishing the printer should be clear that only the fonts supplied by the map maker should be used.

\section{COLOR TRAPPING}

Trapping digital files is the process that compensates for registration on the printing press by printing small areas of overlapping color where objects meet. The concept of trapping arises from the physics surrounding the printing process and includes: taking 4 or more plates which all contain portions or colors of the same image (file); mounting them on a printing press sometimes 80 feet long, that has high-speed spinning cylinders; printing images one on top of the other on a sheet of unstable and extremely thin paper sometimes 3 feet wide or more; and applying ink, water, and chemicals to these plates and paper at speeds exceeding $50 \mathrm{mph}$. Everything on the final, printed sheet must look sharp, appealing, in focus to the eye, and be correctly registered.

Trapping can be accomplished either manually or by means of an automated process. Most graphics or illustration software packages and page layout software offer some kind of automated trapping functionality. Printers and service providers may also have dedicated software systems for trapping. Depending on the effect that is needed, trapping for maps may be best accomplished manually. For instance a trap of 0.002 inches will allow for compensation of any physical misregistration, while prohibiting the appearance of "keylines".

Trapping is going to produce process and media specific differences, so an effective trapping strategy for one map may not suffice for another map. The experience of the printer, cartographer, and even environmental conditions such as the temperature and humidity of the pressroom can impact the effect of trapping. In RIP Printers automatic trapping is global, creating a relationship between touching colors. The Printer can manually trap in the RIP program, if required. Whether a map requires trapping manually or by an automatic process, the cartographer should always check with the printer to verify the trapping settings.

All this said, it is possible to avoid the need for trapping. The first strategy is to not touch the colors or to use process colors in any way. This is almost always impractical because of design limitations.

A second method is knockout and overprint. Overprint is where one color is printed on top

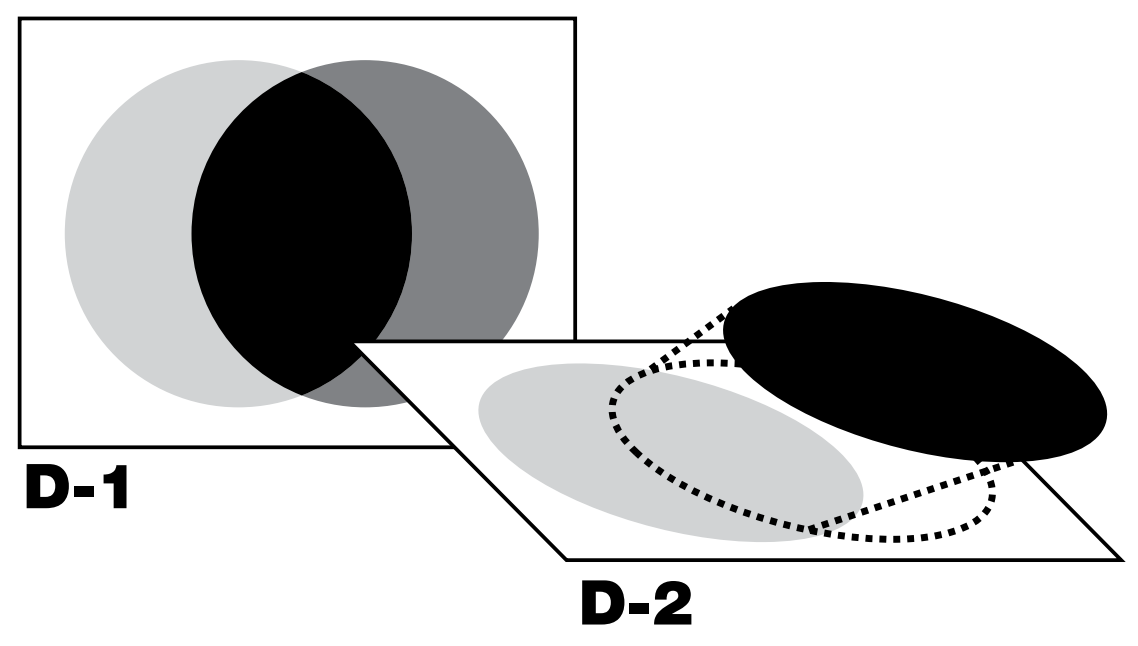

Figure 1. Note that the yellow circle isn't really a circle underneath it all. (See page 63 for color version) 
of another color, resulting in an additive color. This is not desired at times. Note the color created where the black overprints the yellow in the upper left portion of Figure 1. Even when using a customer's application trapping setting, the printer may choose to globally overprint black automatically. You can anticipate this, and compensate by tricking the printers software by making your blacks that must not overprinted 100-k 1-c 1-m 1-y. Make heavy solid black areas $100-\mathrm{k} 40-\mathrm{c}$ for a deep rich black. Trap these as a normal color. Knockout-one color 'knocks out' the color underneath, but a trap is required.

Sometimes projects require special trapping regimes in order to be properly executed. If the cartographer has a full understanding of trapping, manually trapping in the applications can give desired results better than automatic trapping, but it will be time-consuming.

\section{CONCLUSIONS}

Knowledge is the key to working successfully with printers and prepress departments. This article has hopefully shown some of the common practices and best methods for cartographers to use when working with a printer to publish a map. However, this article is really the tip of the proverbial iceberg. Map makers should educate themselves prior to designing their map and especially prior to visiting their printer. Check out trade publications that publish techniques such as The Journal of Electronic Publishing, American Printer magazine, High Volume Printing, Graphic Arts Monthly, and Newspapers and Technology. For working successfully in color managed environments consult reference books such as the GATF Practical Guide to Color Managment and Understanding Color Management by Abhay Sharma.
There are also online groups such as colortheory@yahoogroups.com and colorsync-users-request@lists. apple.com.

\section{REFERENCES}

Burnett M. 2004. Working With Your Printer. Cartographic Perspectives No. 49, Fall 2004.

Adobe Systems Inc. 1993. Adobe Type 1 Font Format. Addison-Wesley Publishing Company, Inc. $3^{\text {rd }}$ Printing. New York. http:// partners.adobe.com/asn/developer/ $\mathrm{pdfs} / \mathrm{tn} / \mathrm{T} 1 \mathrm{SPEC} . \mathrm{PDF}$

\section{map library bulletin board}

\section{Penn State University Libraries: Building a Literary Map for the Web}

By Joanne M. Perry,

Maps Librarian

With technical assistance from Derrick Beckner and Karen Schwentner

The Pennsylvania State University, Universities Library

In late summer 2000, Dr. Steven Herb, the Penn State Education Librarian and Director of the Pennsylvania Center for the Book, asked if the Maps Library would collaborate on a mapping project. Dr. Herb wanted to provide the Pennsylvania citizenry, the schools, and public libraries with access to information about Pennsylvania authors and their books in the form of a Web-based interactive map with an integrated biographical database. In other words, he wanted to design an on-line literary map. Intrigued, I agreed to assign the Maps Li- brary's GIS support staff member, Derrick Beckner, to design the base map.

The original Literary Map of Pennsylvania was published by the Pennsylvania Council of Teachers of English in 1959, and revised in 1965 . After nearly 40 years, Dr. Herb felt that Pennsylvania's many noted authors deserved a revised map with more authors and a greater depth of information, and he believed that the online environment was capable of delivering a much improved product.

The decision to develop an online map instead of another paper revision was both cultural and financial. The Web is where exciting things happen these days and, being practical, it avoids printing, storage, and shipping costs in the delivery of the product to the user. While the development costs of Web products are substantial, the Web also permits the use of animation, color is no more ex-pensive than black and white, and more information can be attached to online maps than can be printed on traditional paper maps. Thus, for a non-profit group or institution, the Web provides a way to transfer information to their constituents relatively economically and efficiently.

The Pennsylvania Center for the Book has a very small parttime staff, so developing the online Literary Map of Pennsylvania has been a cooperative venture. Sixty people at the University Park campus (5 part-time paid workers, 5 part-time interns, 8 Journalism interns, 1 Independent Studies student, 40 English students, 1 Maps Library staff member, 2 Public Relations staff members, and 1 computer technician) as well as public librarians and county historians from each of the sixty-seven counties have provided support during the development period.

In designing the literary map, Derrick Beckner used Adobe Il- 